\title{
A pictorial review of acute aortic syndrome: discriminating and overlapping features as revealed by ECG-gated multidetector-row CT angiography
}

\author{
Takuya Ueda • Anne Chin • Ivan Petrovitch • \\ Dominik Fleischmann
}

Received: 5 August 2012 /Revised: 14 September 2012 / Accepted: 19 September 2012 / Published online: 6 November 2012

(C) The Author(s) 2012. This article is published with open access at Springerlink.com

\begin{abstract}
Background The term "acute aortic syndrome" (AAS) encompasses a spectrum of life-threatening conditions characterized by acute aortic pain. AAS traditionally embraces three abnormalities including classic aortic dissection, intramural haematoma, and penetrating atherosclerotic ulcer. Although the underlying etiologies and conditions predisposing to AAS are diverse, the clinical features are indistinguishable.

Methods Multidetector-row computed tomography (CT) with electrocardiographic gating (ECG-gated MDCT) has greatly improved imaging of acute thoracic aortic diseases by virtually eliminating pulsation artifacts transmitted from cardiac motion and reveals subtle aortic abnormalities, which have been difficult to recognize by conventional non-gated CT.
\end{abstract}

This study was performed at the Department of Radiology, Stanford University School of Medicine, Stanford, CA.

T. Ueda $(\bowtie)$

Department of Radiology, St. Luke's International Hospital,

9-1 Akashi-cho,

Chuo-ku, Tokyo 104-8560, Japan

e-mail: takueda@luke.or.jp

A. Chin

Department of Radiology,

Centre Hospitalier de L'Univeristé de Montréal,

3480 rue St. Urbain,

Montreal, Quebec, Canada

I. Petrovitch

Northern Virginia Radiology Consultants, PLLC,

1701 N. George Mason Drive,

Arlington, VA 22205, USA

D. Fleischmann

Department of Radiology,

Stanford University School of Medicine,

300 Pasteur Drive,

Stanford, CA 94305, USA
Results While these advances in imaging technology provide additional discriminating features of acute aortic diseases, they also reveal a range of overlapping features of these life-threatening conditions that not uncommonly are dynamic and evolving. These overlapping and transitional features may be a major source of misunderstanding, confusion, and controversy for diseases that cause AAS.

Conclusion In this pictorial review, we describe the discriminating and typical imaging features as revealed by modern ECG-gated MDCT angiography. In addition to the discriminating features, recognition of the overlapping and transitional features in AAS will allow a more comprehensive understanding of their underlying pathophysiologic conditions and their natural history, and may improve therapeutic management.

Main Messages

- The superior visualization of ECG-gated CTA improves the diagnostic accuracy of acute aortic syndrome.

- ECG-gated CTA provides discriminating features of underlying pathophysiologic conditions of AAS.

- Also, recognition of the overlapping features in AAS will allow a more comprehensive understanding.

Keywords Aortic diseases · Tomography $\cdot$ X-ray computed $\cdot$ Aneurysm $\cdot$ Aortic dissection $\cdot$ Aortic aneurysm

\section{Introduction}

Acute aortic syndrome

The term 'acute aortic syndrome' (AAS) refers to a spectrum of acute, life-threatening conditions of aortic diseases characterized clinically by abrupt, intense chest and/or back pain [1]. AAS traditionally embraces three aortic diseases: aortic dissection (AD), intramural haematoma (IMH) and 
penetrating atherosclerotic ulcer (PAU) $[1,2]$. The differentiation of disease entities in AAS is essential for predicting the natural history and for initiating the appropriate treatment in a timely fashion [2,3]. However, the presenting clinical signs and symptoms of AAS of any etiology are usually clinically indistinguishable [4]. Although typical cases demonstrate characteristic imaging features of each disease, imaging findings may also overlap between different entities, especially when the process is dynamic and evolving. These transitional and overlapping features of AAS, both clinical and pertaining to imaging findings, have led to several misconceptions and controversies concerning the disease concept of AAS.

CT angiography (CTA) is an established imaging technique to diagnose $\mathrm{AAS}$ in the emergency setting, and newer generation multidetector-row CT (MDCT) scanners have improved the sensitivities and specificities of aortic pathologies [5]. Additionally, the introduction of the retrospective or prospective electrocardiographic (ECG)-gated technique has virtually eliminated the notorious pulsation artifacts in the ascending aorta and allows for a substantially improved assessment of subtle aortic abnormalities previously underrecognized by non-gated CTA (Fig. 1) [6]. The superior visualization of ECG-gated CTA dramatically improves the detectability of some pathognomonic findings such as the primary intimal tear in thrombosed $\mathrm{AD}$ or ulcerative plaque in PAU $[2,6]$.

In this pictorial review, we illustrate discriminating imaging features of AAS disease entities as well as the overlapping and transitional ones as revealed by modern ECGgated CTA (Fig. 2). Awareness of some related and distinguishing radiologic features in AAS may improve our understanding of these diseases and provide further insight into the pathophysiology and natural history and guide appropriate management of these lesions.

\section{Aortic dissection}

Classic aortic dissection (AD) is characterized by separation of the layers of the aortic media initiated by a primary intimal tear, with variable longitudinal and circumferential extension, resulting in two distinct flow channels: the true lumen, contained entirely by the intima, and the false lumen, contained entirely within the medial layer of the aorta (Fig. 3) [7]. Degeneration of the medial layer of the aortic wall is the fundamental pathophysiology of AD [8]. The common pathologic denominator (process) resulting in medial degeneration, initially termed 'cystic media necrosis' by Erdheim in 1929, has histologic features characterized by non-inflammatory loss of vascular smooth muscle cells and elastolysis of aortic wall components [9]. 'Cystic media necrosis' is the common end path of various acquired and genetic conditions weakening the medial layers of the aorta leading to the breakdown in the integrity of the aortic wall, which precedes AD [10]. The most common comorbid condition for cystic media necrosis and AD is severe arterial hypertension. Chronic exposure of the aorta to high blood pressure may induce medial disruption and degeneration of the aortic wall [8]. In addition, several genetic conditionssuch as Marfan syndrome, Ehlers-Danlos syndrome and familial aortic aneurysm/dissection - are well-known precursors of AD as well [11]. Intimal disease - namely atherosclerosis - is not a prerequisite for $\mathrm{AD}$, although it may coexist particularly in the elderly. This is in contradistinction to PAU, which is essentially a manifestation of severe and advanced atherosclerosis [12].

Two anatomical systems - the DeBarkey and Stanford classification-are used to classify aortic dissection [2, 10]. Recently, the Stanford system has been more widely used worldwide as it is simpler and directly connected with the therapeutic strategy. Stanford type A dissection, which involves the ascending aorta regardless of the site of the primary intimal tear, should be treated as a surgical emergency as these patients are at high risk for a life-threatening complication with a high mortality rate $(1-2 \%$ per hour early after symptom onset) $[2,10]$. Patients with uncomplicated Stanford type B dissection, which was confined to the aortic arch and the descending aorta, are best treated with medical therapy; the in-hospital mortality for these patients was $10 \%[2,10]$. Radiological imaging plays an essential role in determination of the therapeutic strategy.

Discriminating and overlapping features of aortic dissection

Classic AD can be unambiguously differentiated from other forms of AAS by the presence of a double-barrel lumen, consisting of a true lumen and a false lumen, resulting from a primary intimal tear, which is usually clearly visualized with state-of-the-art CT technology: the false lumen is contained entirely within the aortic media and is separated from the true lumen by a dissection flap or 'intimal flap.' However, confusion with respect to the terminology arises when classic $\mathrm{AD}$ is associated with non-flowing blood or haemorrhagic content within the medial layer. This is not uncommon in situations when the primary intimal tear is more distal (e.g., in the descending aorta) than the most proximal extent of the medial separation (which may extend retrogradely into the ascending aorta), where stagnating blood or clots can be observed. In CT, stagnating blood in the false lumen is isodense to blood within the true lumen, whereas thrombosed blood is hyperdense relative to flowing blood in non-contrast CT. The extreme within the spectrum of separation of medial layers as a consequence of underlying 'cystic media necrosis' occurs in complete thrombosis of the false lumen, resulting in the imaging features of IMH, 
Fig. 1 Impact of ECG-gated CT angiography (CTA). Nongated $(\mathbf{a}, \mathbf{b})$ and ECG-gated (c, d) CTA in a 66-year-old man with limited intimal tear. a Nongated axial CT image shows a 'dissection-like' motion artifact (arrow) on the contour of the aorta because of pulsatile motion of the aorta. b Oblique sagittal slab maximum intensity projection (slab-MIP) image shows an undulating contour of the aorta caused by misregistration from the pulsatile motion. c ECG-gated CTA, performed 1 day after the nongated CT, eliminates the pulsatile motion artifact. The image clearly reveals a hidden focal intimal tear (arrowhead). d Slab MIP image of ECG-gated CTA demonstrates visualization of the ascending aorta without misregistration artifacts. A focal bulge with a tiny intimal tear is revealed (curved arrow)
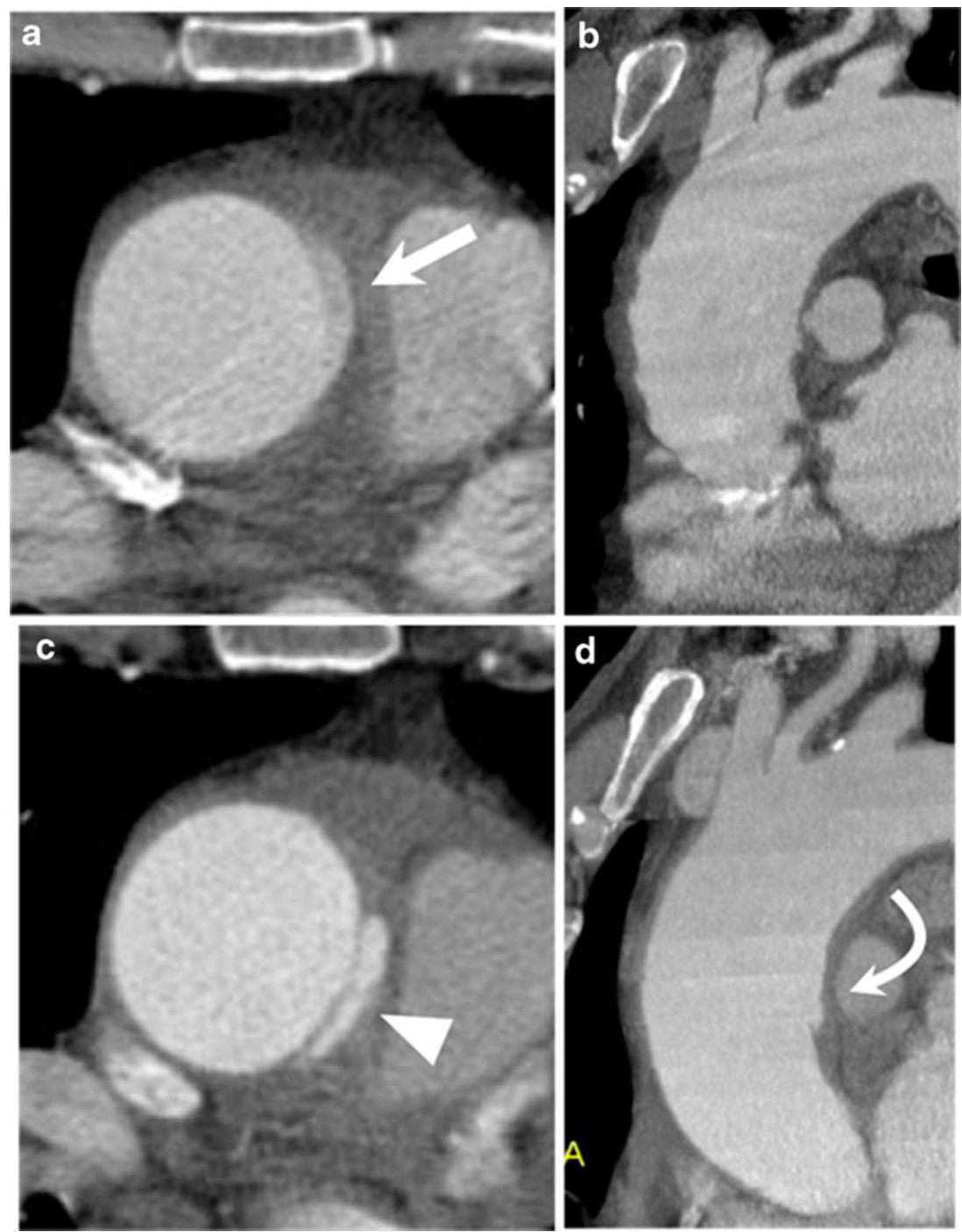

and in this context, IMH is considered a variant of classic AD. Another variant of AD-called limited intimal tear or discrete/subtle tear-is characterized by a complex primary intimal tear with only minimal separation between the medial layers at the edges of the lesion; this disease entity also has a pathophysiology and etiology overlapping with classic dissection [9, 13, 14]. These topics are elaborated below.

\section{Intramural haematoma}

Intramural haematoma is defined as a hyperdense, crescentshaped lesion within the aortic wall on non-enhanced CT (Fig. 4) [15, 16]. As defined originally, IMH has no demonstrable intimal flap or radiologically apparent intimal tear [17]. This view is outdated, however, since modern imaging technology can usually detect small communications between the true and false lumen [2].
The etiology of IMH remains controversial [17]. Initially, IMH was considered as a distinct independent disease manifestation that arises from haemorrhage of the vasa vasorum of the aorta [18]. Increasingly, many authors have reported an overlap between classic AD and IMH [19, 20] and considered that the haematoma results from microscopic tears in the aortic intima [2]. Focal IMH has also been reported in association with PAU [20]. This widely propagated notion has resulted in a 'classification' of AAS that is used almost exclusively in the literature, where IMH is categorized as a separate 'disease manifestation' along with $\mathrm{AD}$ and PAU [1]. Adding to the confusion, the term IMH has been used inconsistently in the literature referring to a 'disease' equivalent, a variant of acute $\mathrm{AD}$, and PAU.

Currently, it is accepted that IMH can be a variant or a precursor of $\mathrm{AD}$, and many have regarded $\mathrm{IMH}$ as synonymous with a 'thrombosed type' or 'non-communicating' AD (Fig. 4) [15, 21] where separation of the medial layers still occurs, but this is filled with thrombus rather than flowing 


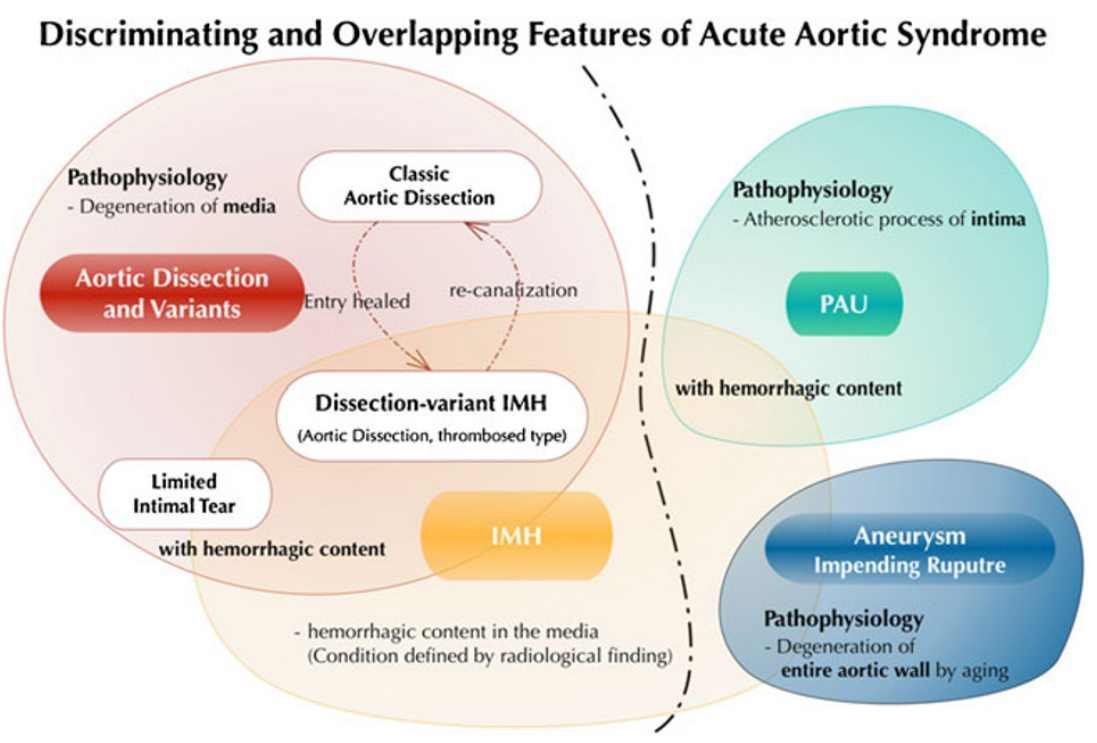

Fig. 2 Discriminating and overlapping features of acute aortic syndrome. In addition to the certain discriminating features as revealed by recent advances in imaging, each disease in acute aortic syndrome (AAS) may show overlapping features and transition from one to another. In such overlapping and transitional features of aortic diseases, the diagnosis may be affected by the time point at which the imaging has been examined and also by the detectability of tiny pathology by the imaging modality. Awareness of some related and distinguishing radiologic features in AAS is helpful in understanding these diseases and providing new insight into the pathophysiology blood in what would otherwise be the false lumen of a classic dissection. On initial imaging, like at the follow-up imaging of IMH, small 'ulcer-like projections' (ULP) - defined as a localized blood-filled pouch protruding from the
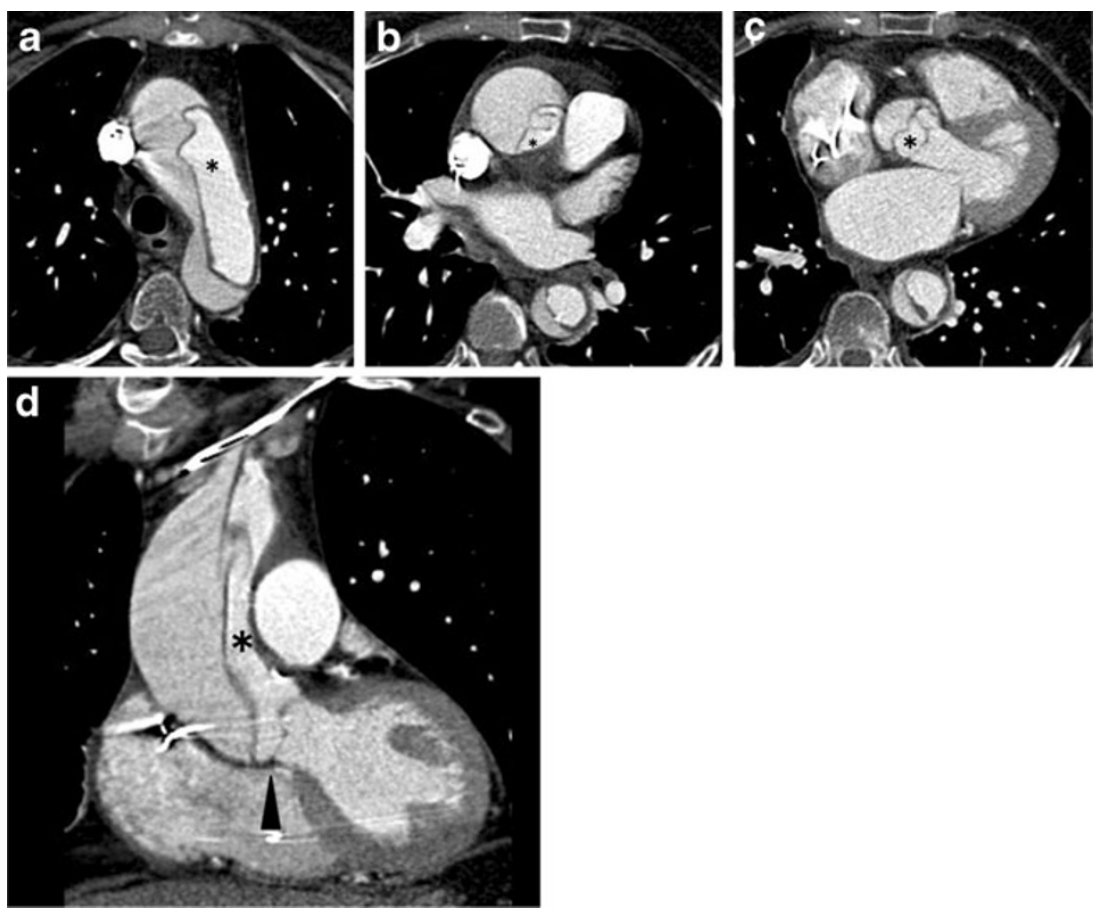

Fig. 3 Acute Stanford type A classic aortic dissection in a 71-year-old woman with abrupt, severe retrosternal chest pain. a-c Transverse images from an ECG-gated CTA demonstrate an extensive intimomedial flap involving the entire thoracic aorta. The true lumen $(*)$ appears small in the ascending aorta where it is nearly circumferentially separated from the rest of the aortic wall (b). Extension of the flap down to the aortic root to involve the aortic valve apparatus is clearly

demonstrated in this patient who had aortic regurgitation confirmed on echocardiography, a significant complication of acute dissection (c). d Multiplanar reformations in a coronal plane show the involvement of the brachiocephalic artery to better advantage (arrow), the near circumferential separation of the aortic media at the level of the ascending aorta and its extension down to the aortic valve apparatus (arrowhead) 


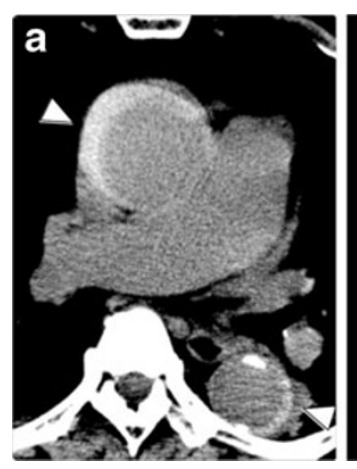

Fig. 4 Dissection variant intramural haematoma (IMH) in a 54-yearold man who presented with acute chest pain. a Non-contrast CT image shows a crescent-shaped high-density area in the ascending aorta and descending aorta (arrowhead). b On the axial contrast-enhanced CT image, neither the flow channel nor intimal flap is detected along the entire aorta, consistent with IMH. c Oblique sagittal MPR image

true lumen into the thrombosed false lumen of the aortacan be observed [22]. In this context, the finding is considered to represent the site of an intimal disruption and is therefore a possible indicator of the formation of a flow channel between the true and thrombosed false lumen and hence evolves into classic AD. The term 'ulcer-like lesion,' however, is non-specific and has been used in the literature to include also non-penetrating as well as chronic ulcers of the aortic wall. In order to avoid confusion, we advocate using the term 'primary intimal tear (PIT)' for an intimal disruption in patients with the dissection variant IMH rather than using 'ULP.' The superior visualization of ECG-gated CTA dramatically improves the sensitivity for detecting small communications between the true and false lumen (PIT) in IMH (Fig. 4), which seems to be the elusive link between classic $\mathrm{AD}$ and IMH $[6,22]$. In fact, a recent autopsy series showed that only $4 \%$ of IMHs have no visible intimal tear; indeed, at the time of surgery a tear is found in most patients [7].

\section{Overlapping and transitional features of IMH}

As we discussed above, it is well known that IMH may be a precursor of and evolve into classic dissection [13]. The prevalence of IMH in AAS has been reported to be $10 \%$ to $50 \%$, and the prevalence in the recent studies reported less than previously thought $[17,23]$. Many initially diagnosed IMHs may develop into classic AD [19, 24]. On the other hand, focal intimal injury (PIT) in IMH may heal and resolve (Fig. 5) [25]. Therefore, it is important to recognize that the imaging features may vary depending on the time period during the course of the disease as the pathological process underlying classic $\mathrm{AD}$ and $\mathrm{IMH}$ is intrinsically overlapped.

In addition to dissection variant IMH, a wide range of acute aortic lesions may demonstrate haemorrhagic content within the aortic wall to variable degrees, most importantly demonstrates widespread extension of type A IMH throughout the whole thoracic aorta. An 'ulcer-like projection,' the radiological finding of a focal disruption of the surface of the aortic wall from the true lumen into the thrombosed false lumen, is identified at the mid ascending aorta (arrow). During surgery, a small thrombosed focal primary intimal tear (PIT) was identified at the corresponding site

PAU, discussed below, but also iatrogenic ADs, traumatic injuries and rupturing aneurysms, etc. In this broad sense IMH can be regarded simply as a non-specific imaging finding, and in fact, cases that were previously categorized as IMH often included a spectrum of unrelated underlying aortic conditions. In order to avoid confusion and misunderstanding, we use the term 'dissection variant IMH' for a thrombosed $\mathrm{AD}$ that has no complete flow channel, but tiny communications between the true and false lumen commonly exist and clearly show differentiation from other aortic diseases with haemorrhagic content within the aortic wall.

\section{Limited intimal tear}

\section{Definition}

A limited intimal tear (also known as 'incomplete tear' or 'subtle/discrete dissection') is a rare variant of AD that is the least recognized disease manifestation of AAS $[13,14]$. Pathologic descriptions of this type of lesion can be found as early as 1930 by Erheim [9], who used the term 'nontraumatic laceration.' Although the incidence of limited intimal tears is not well known and is likely underestimated because of general unfamiliarity with this dissection variant [14], the European Society of Cardiology included this lesion in its classification of dissection subtypes (subtle/discrete dissection, class 3) [21], which was reiterated in the guideline of the American College of Cardiology Foundation and the American Heart Association in 2010 [2]. The limited intimal tear is a subtype of AD that is characterized pathologically by a stellate or linear tear through the intima and underlying superficial media that results in exposure of the deeper media or adventitial layers [13]. The intimal tear extends to a variable degree within the aortic media without significant separation of the medial layers and does not result in a second flow channel, as seen in classic $\mathrm{AD}$ [13]. 
Fig. 5 Resolved tiny primary intimal tear of dissection variant IMH in a 68-year-old woman with hypertension who presented with unstable thoracoabdominal pain. a Non-contrast CT image shows a crescentshaped high-density area in the descending aorta (arrowhead). b Slab MIP image demonstrates haematoma extending through the whole thoracic descending aorta, which consists of dissection variant IMH. A tiny PIT is detected at the distal end of the haematoma (arrow). c The patient was followed with stable limited chest pain. After 7-day follow-up, the PIT resolved spontaneously, leaving a slightly enlarged haematoma (arrow). The patient was treated conservatively, and the haematoma eventually resolved
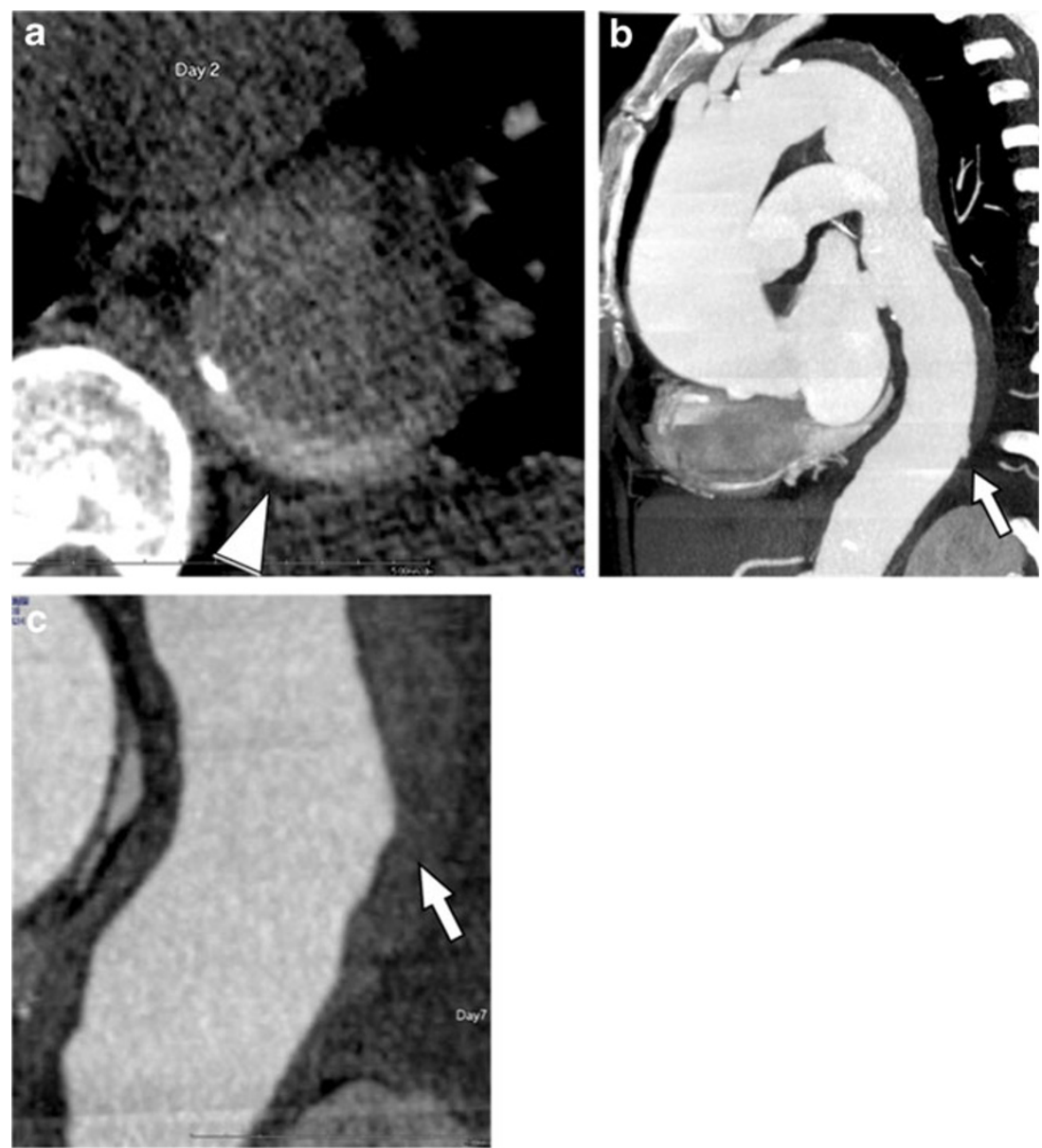

A limited intimal tear is particularly difficult to visualize with traditional imaging modalities [13]. The longitudinal tear on the intimal superficial surface is difficult to detect by most imaging modalities, although Chirillo et al. have reported the benefit of transesophageal echocardiography in detecting the intimal abnormality [14]. The eccentric bulge may be the only imaging finding of this lesion, which can be easily overlooked with traditional imaging modalities $[13,14]$. Currently, ECG-gated CTA can have potential utility in detecting subtle forms of limited intimal tear [6]. In our experiences, limited intimal tear demonstrated a minor contour abnormality of the aortic wall ('eccentric onesided bulge') on ECG-gated CTA (Fig. 6). Eccentric onesided bulges are sometimes accompanied by haemorrhagic content within the aortic wall on non-contrast CT imaging and subtle undermined edges on CTA (Fig. 6). Advanced post-processing techniques - particularly 3D-VR and virtual luminal views - can greatly increase the conspicuity of these often extremely subtle limited intimal tears (Fig. 6) [6].

Discriminating and overlapping features of limited intimal tear

Previously, limited intimal tears might have been diagnosed as other aortic diseases in AAS or missed all together because of the difficulty in detecting this discrete finding with standard imaging modalities [13, 26, 27]. As limited intimal tear remains an unfamiliar entity, the focal bulge associated with this lesion may be incorrectly diagnosed as either an atherosclerotic aneurysm or pseudoaneurysm. As a limited intimal tear and classic AD share the same pathophysiologic process - a weakened medial layer of the aortic wall - these two dissection subtypes may, not unexpectedly, show overlapping features. We have experienced some cases where an initial focal intimal tear evolves into a classic AD locally to form true and false lumens (Fig. 6). Overlapping imaging of features of IMH can also occur as limited intimal tears may also demonstrate a small amount of haemorrhagic content surrounding the tear within the aortic media (Fig. 6) [28].

\section{Penetrating atherosclerotic ulcer (PAU)}

PAU is defined as an atherosclerotic ulceration that penetrates from the pathologically thickened intima through the internal elastic lamina into the media of the aortic wall [12]. PAU is a manifestation of advanced atherosclerosis and therefore a manifestation of a diseased intima (and not the 
Fig. 6 Limited intimal tear with intramural haemorrhagic content in a 48-year-old male. a Non-contrast CT demonstrates the aortic wall thickening with haemorrhagic content (arrow) at the proximal aortic arch. b Contrast-enhanced CTA demonstrates the aortic wall thickening (arrow) without clear intimal-medial flap or false lumen visualized; this was initially diagnosed as traditional IMH. c Slab-MIP image demonstrates the "eccentric onesided bulge' of the aortic wall along the ascending aorta (arrowheads). d 3D-VR luminal image right in front of the bulge shows a teardrop-shaped intimal tear (dotted line) with a localized intimal flap (black arrows) at the inferior border of the intimal tear
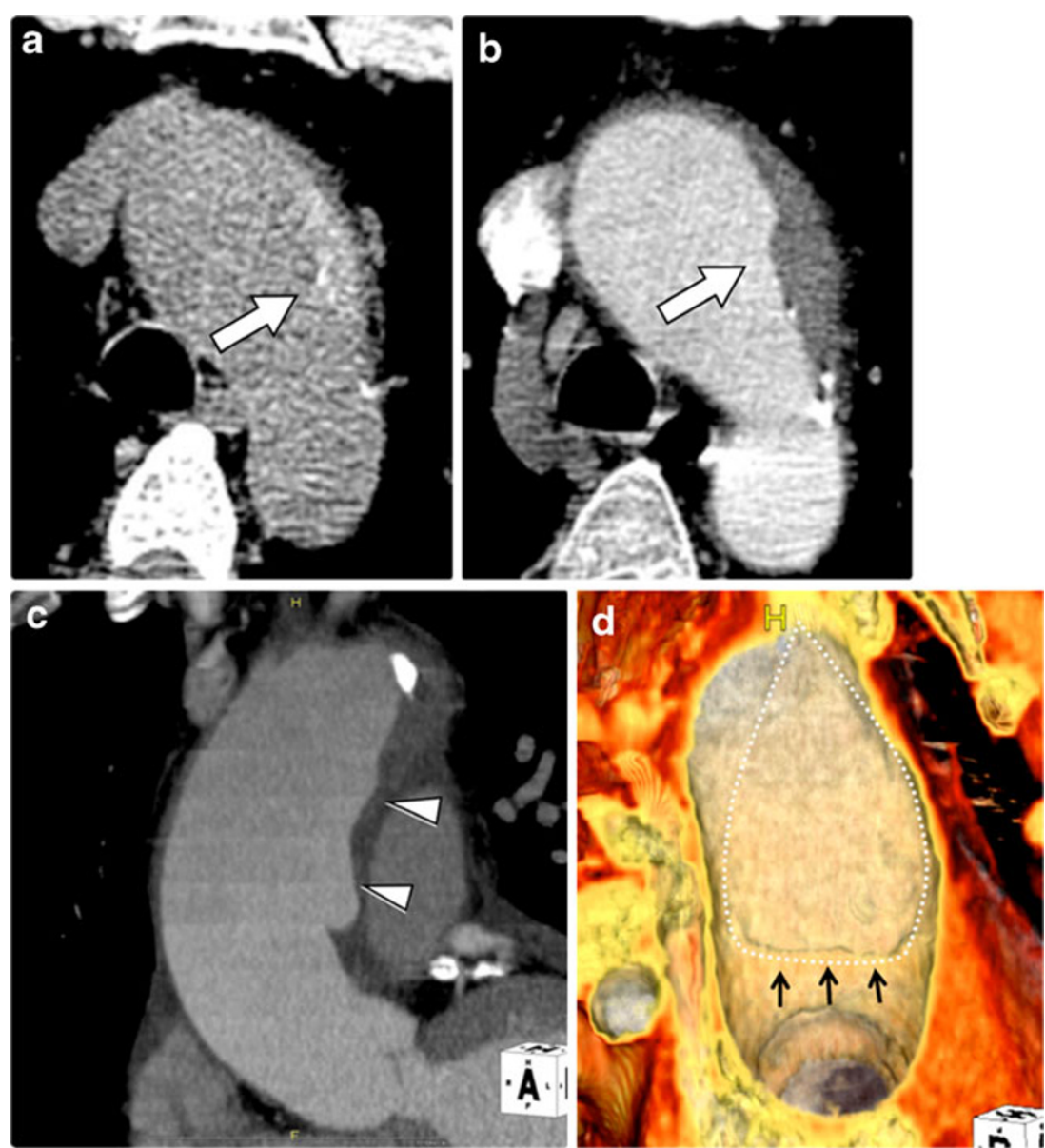

media, as in $\mathrm{AD}$ ) [12]. The lesion may penetrate even beyond the media and extend through to the adventitia, producing a periaortic pseudoaneurysm and even transmural aortic rupture [12].

It is important to appreciate the fundamental distinction in the underlying pathological processes between PAU and AD [29]. PAU occurs in the setting of severely and extensively diseased intima in patients with advanced atherosclerosis. Clinically, the typical profile of a patient with PAU is an elderly individual with multiple risk factors for atherosclerosis and often already documented manifestation of atherosclerotic disease, such as coronary aortic disease, cardiovascular disease, peripheral arterial disease or abdominal aortic aneurysm [13]. Patients with $\mathrm{AD}$, on the other hand, are relatively younger and besides hypertension may not have any other risk factors for atherosclerosis $[10,12,30]$.

Differentiation between PAU and a dissection variant IMH is clinically important as the prognosis and management differ depending on the underlying disease entity. PAUs arise in segments of the aorta where atherosclerotic changes are more common, and therefore over $90 \%$ of PAUs are localized in the descending thoracic aorta [31].
Nevertheless, irrespective of their location, PAUs tend to have a bad prognosis - even when disease is limited to the descending aorta - with a higher incidence of aortic rupture compared with aortic dissection. For this reason, some authors advocate a more aggressive approach with endovascular treatment for acutely symptomatic PAUs in the descending aorta, as an open surgical approach is generally prohibitively risky in these patients with multiple comorbidities [16]. This is in contrast to acute type B dissection variant IMH or $\mathrm{AD}$, which tends to be more stable and respond well to aggressive medical management. The less common PAU in the ascending aorta should also be managed surgically, like any acute type A aortic lesion.

\section{Discriminating and overlapping features of PAU}

On imaging, patients with typical PAU invariably demonstrate extensive atheroma and calcification throughout the thoracic and abdominal aorta, often with an irregular surface (Figs. 7 and 8) [12]. Sundt et al. reported findings of PAU on transesophageal echocardiography, including a crater-like ulcer with jagged edges [29]. In our experience, PAUs commonly show a 'crater-like ulceration' of thickened 
Fig. 7 Penetrating

atherosclerotic ulcer with haemorrhagic content in a 74year-old man. a Non-contrast CT shows intramural haemorrhagic content (arrow) in the descending aorta. Note the thick calcification and plaque on the aortic wall $(*)$, suggesting an atherosclerotic background of the patient. b Axial ECG-gated CTA demonstrates the ulcerative lesion at the proximal ascending aorta (arrowhead) into the aortic media, which shows associated haemorrhage. c 3DVR image depicts the craterlike ulceration (arrowhead) and atherosclerotic calcification $(*)$ on the aortic arch. Some nonpenetrating atherosclerotic ulcers are also identified (arrows). The condition is considered as an overlapping feature between IMH (in the broad sense) and penetrating atherosclerotic ulcer
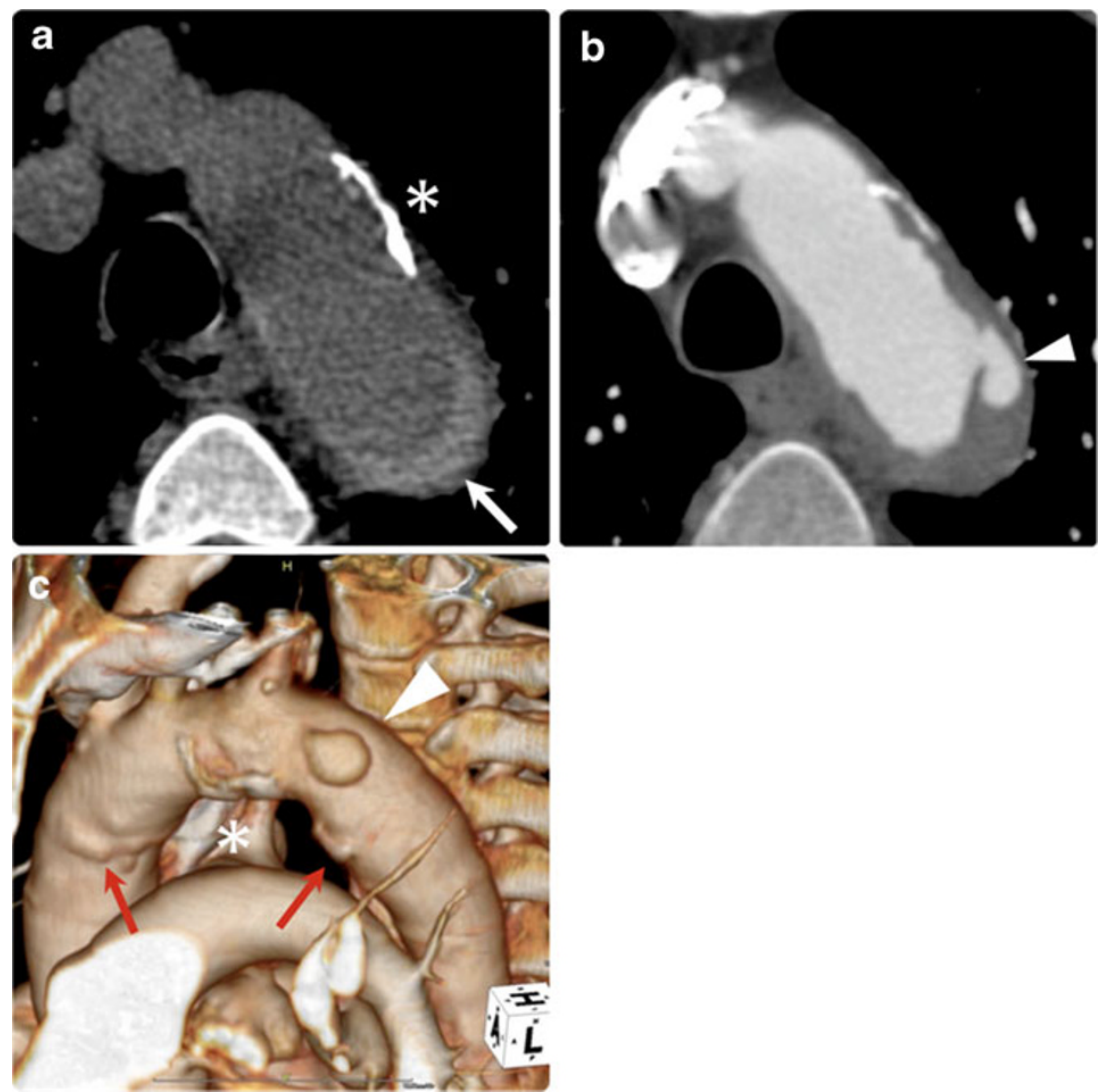

aortic wall on ECG-gated CTA (Figs. 7 and 8), which is different from the linear or crescent shape of PIT in dissection variant IMH.

As the crater-like ulcerations of PAU are often accompanied by haemorrhagic content within the medial layer of the aortic wall [16], non-contrast enhanced CT demonstrates high-density haematoma in the aortic wall surrounding the ulceration (Fig. 9). Because of this accompanying haematoma, in the past, PAU has often been lumped together as 'IMH' with thrombosed AD (dissection variant IMH) as a cause of AAS [29]. Also, such lesions may have been diagnosed as 'traditional IMH' when ulceration was not evident enough in traditional imaging modalities, which caused the etiological confusion and misunderstanding with classic aortic dissection and its variants [16]. As discussed above, IMH is now more appropriately regarded as a variant of $\mathrm{AD}$ $[15,21]$, and its pathogenesis is different from that of PAU. In order to avoid confusion, we recommend that 'PAU with haemorrhagic content' that has an atherosclerotic pathogenesis should be clearly differentiated from the dissection variant IMH. The key to the differential diagnosis is underlying systemic atherosclerosis such as severe calcification of systemic arteries, coexisting cardiovascular diseases, aortoiliac and peripheral arterial occlusive diseases.
Nevertheless, clinical and imaging features of AD and PAU can overlap, especially in older patients, who often have two co-existing pathologic processes (i.e., severe atheroslceorsis, as well as 'cystic media necrosis,' which is also seen as part of normal aging). Elderly patients with many comorbidities may possess risk factors that indicate a predisposition to both atherosclerosis and AD simultaneously. In such patients, the differentiation between a limited intimal tear in AD and small atherosclerotic ulcer is sometimes difficult or impossible. In any case, the presence of acute haemorrhage in the aortic wall represents an aortic emergency irrespective of etiology.

\section{Impending rupture of aortic aneurysms}

Thoracic aortic aneurysms (TAA) are often clinically asymptomatic and gradually increase in size over time. Although relatively uncommon, TAAs may rapidly increase in size, with increased risk of rupture (Fig. 9). The risk of rupture is closely associated with aneurysm size: for aneurysms greater than $6 \mathrm{~cm}$ in diameter, the risk of rupture is significantly higher [32]. Elective repair is indicated to prevent rupture. Rupturing TAAs may present as AAS with acute chest pain [33]. Again, the clinical presentation is indistinguishable from AD or PAU [33]. As such, although 


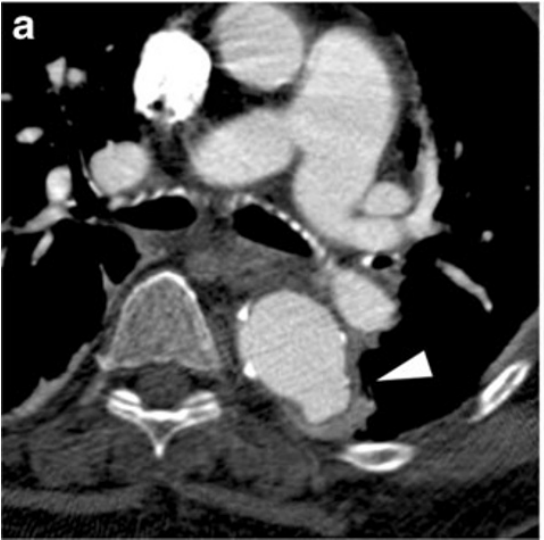

Fig. 8 Penetrating atherosclerotic ulcer in an 81-year-old man. a Axial CTA images demonstrate an ulcerative lesion (arrowhead) on the thickened aortic wall with haemorrhagic content in the descending aorta. b Oblique sagittal MPR image clearly visualizes a crater-like

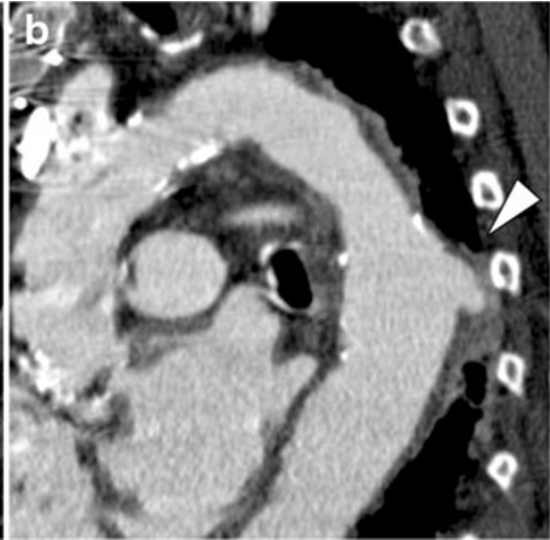

feature of the ulcerative lesion (arrowhead) deeply penetrating into the atherosclerotic aortic wall. Entire aorta depicts severe atherosclerosis with plaque burden and calcifications

conditions still remains. A true aortic aneurysm is defined as dilatation of the aorta that contains all layers of the aortic wall and usually involves the entire circumference [33]. Pathologically, an aneurysm is characterized by deterioration of the aortic wall with loss of elastin and smooth muscle cells [33]. Reed et al. reported the clear etiological difference between degenerative aneurysm and dissection [35]. The most striking difference was the age-specific incidence
Fig. 9 Impending rupture of an atherosclerotic aortic aneurysm in an 83-year-old man. The patient had been followed for stable aortic aneurysm for many years and suddenly presented with acute chest pain. a Noncontrast CT demonstrates ballooning of the aorta at the aortic arch. The aortic wall shows haemorrhagic content within the aortic wall (arrow), which suggests an acute process of this event. b Axial CTA depicts bulged dilatation of the aorta on the right side of the aortic arch (arrowheads), which was not depicted on the previous follow-up CT images. c Oblique coronal MPR image demonstrates dilatation of the aorta (arrowheads) with a thickened aortic wall with haemorrhagic content. The aortic lumen shows an eccentric bulge, a sign of impending rupture of an atherosclerotic aortic aneurysm

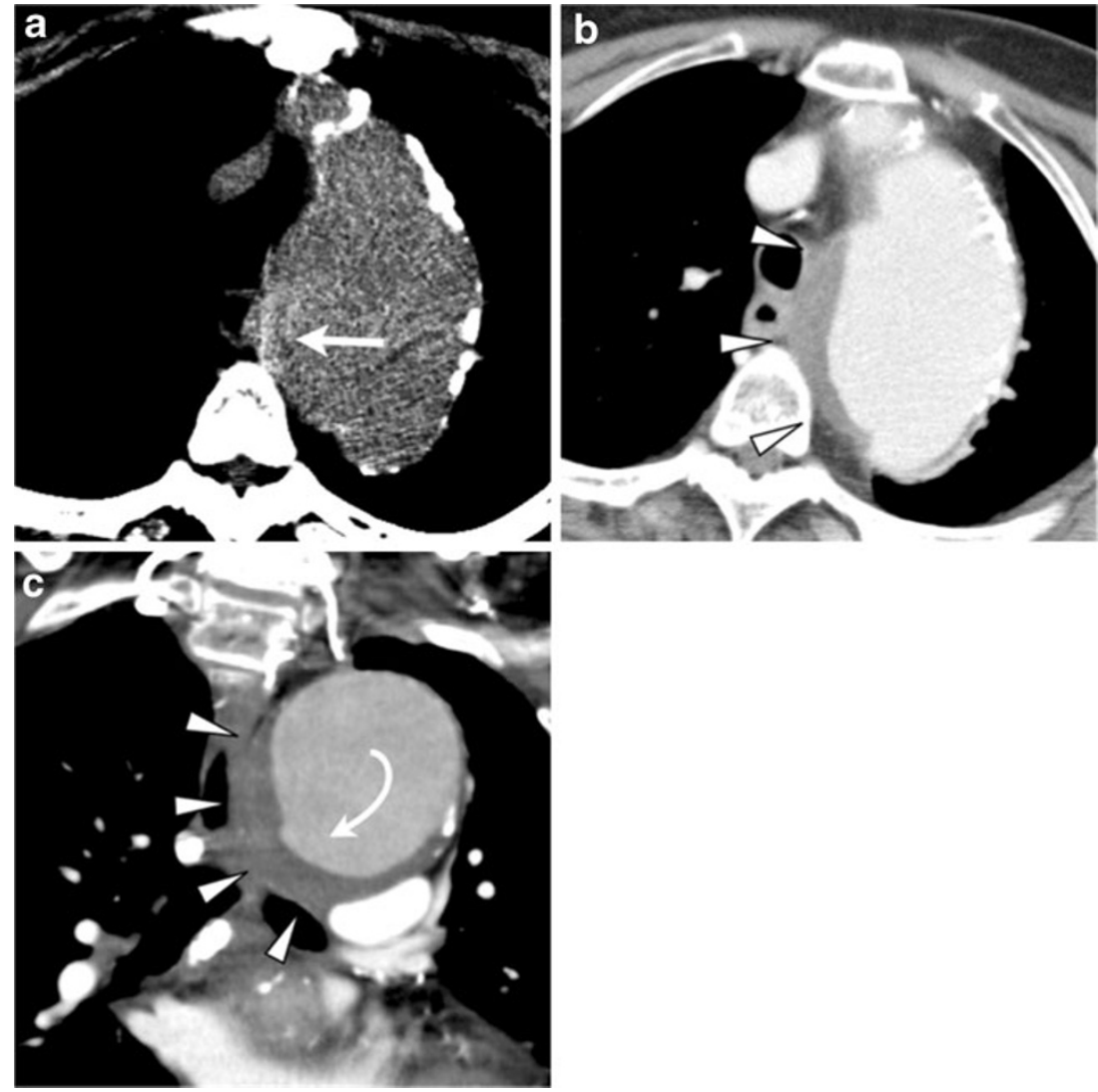


rates: aortic aneurysm increased steadily with 50 years of age, whereas AD peaked around 65 years and decreased thereafter [35]. Degenerative aneurysms are also considered as a manifestation of atherosclerosis.

Atherosclerotic aneurysm vs. aneurysmal dilatation of various aortic pathologies

In addition to degenerative aneurysms, many kinds of aortic pathology may show aneurysmal dilatation in their chronic stage. Chronic AD with associated aneurysmal dilatation of the false lumen can be easily differentiated from standard atherosclerotic aortic aneurysms, even if the intimomedial flap is chronically degenerated, fragmented and partially absent. However, if a dissection variant IMH shows dilatation of the aorta, this may not always be easily differentiated from a true aortic aneurysm, and once the haemorrhagic content is resolved in the subacute and chronic phase, these lesions may be indistinuishable (Fig. 10). Limited intimal tears can heal and may appear as an eccentric aneurysm in the chronic phase and continue to dilate over time (Fig. 6). Chronic PAU may also show aneurysmal dilatation by 'aortic remodeling,' which is usually focal and eccentric [31].

\section{Conclusion}

The conceptual classification of culprit aortic lesions of AAS into pathologies involving either a diseased media (medial degeneration) or a diseased intima (advanced atherosclerosis) avoids some of the confusing terminology regarding AAS. In addition to the discriminating features of each aortic disease, recognition of the overlapping and transitional features in the dynamic and evolving process of AAS will allow a more comprehensive understanding of their underlying pathophysiologic conditions and their natural history, and may improve strategies for therapeutic management. Understanding these features will provide the radiologist with helpful clues

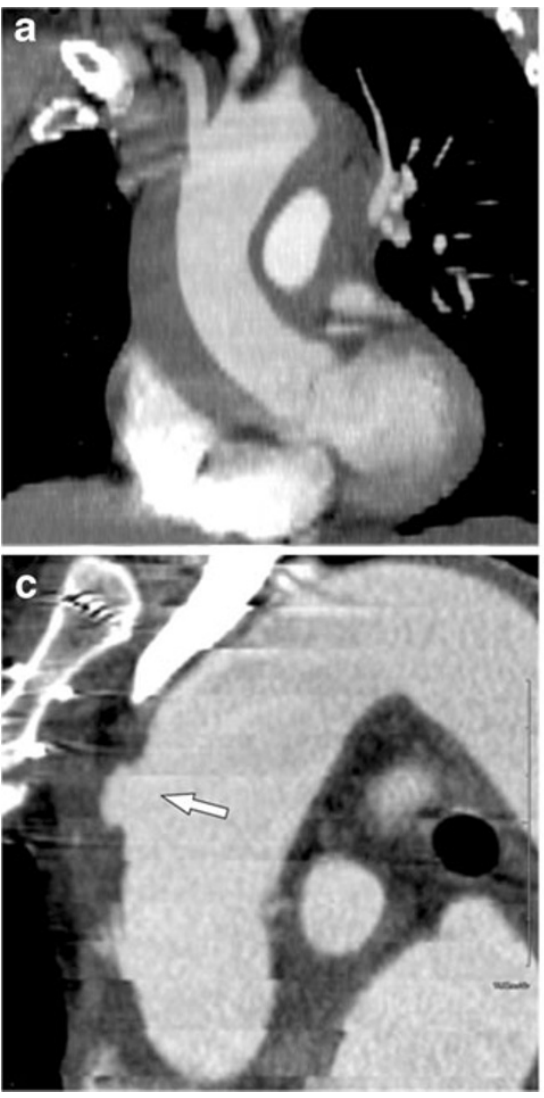

Fig. 10 Aneurysmal dilatation of the aorta secondary to dissection variant IMH in a 66-year-old woman. a Oblique sagittal contrastenhanced CT demonstrates IMH extending from the ascending aorta through the proximal descending aorta. b Axial image depicts a streakform ulcer-like projection (primary intimal tear) in the descending aorta (arrowhead). c Two days after onset, an ulcer-like projection was enlarged to form an outpouching cavity into the thrombosed false lumen (small arrow). d One week later, the haemorrhagic content is

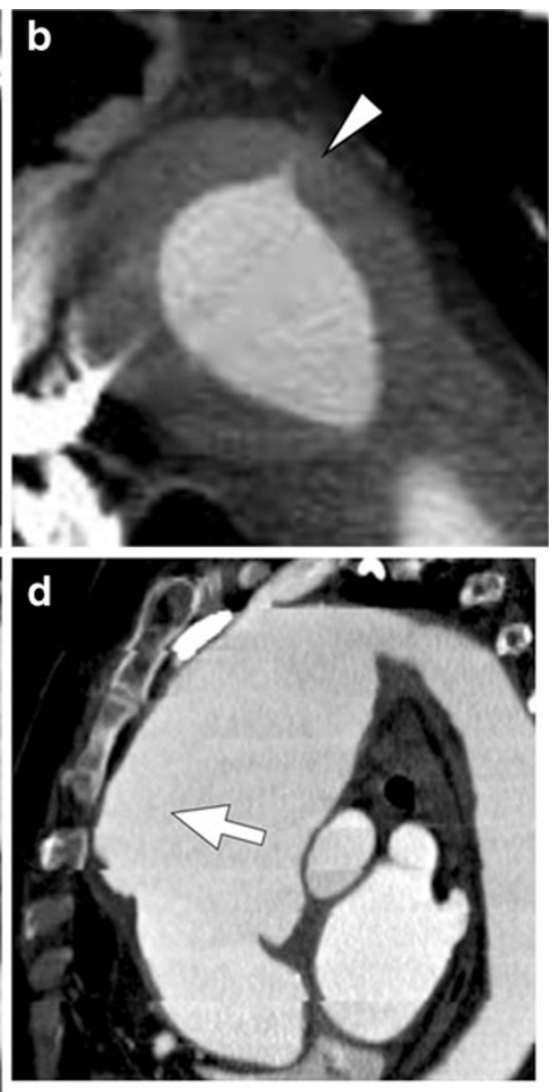

resolved and the ulcer-like projection not been detected. On the other hand, the aorta shows aneurysmal dilatation. Note that the dilatation starts from the location where the haematoma was present (large arrow). Although the pathological features and clinical course are different, such aneurysmal dilatation of dissection variant IMH might be confused with an atherosclerotic aortic aneurysm if an ulcer-like projection is not detected on the initial CT examination 
facilitating image assessment, allowing further prediction of the natural disease course as well as permitting appropriate management recommendations.

Open Access This article is distributed under the terms of the Creative Commons Attribution License which permits any use, distribution, and reproduction in any medium, provided the original author(s) and the source are credited.

\section{References}

1. Vilacosta I, Roman JA (2001) Acute aortic syndrome. Heart $85: 365-368$

2. Hiratzka LF, Bakris GL, Beckman JA et al (2010) ACCF/AHA/ AATS/ACR/ASA/SCA/SCAI/ SIR/STS/SVM guidelines for the diagnosis and management of patients with Thoracic Aortic Disease: a report of the American College of Cardiology Foundation/ American Heart Association Task Force on Practice Guidelines, American Association for Thoracic Surgery, American College of Radiology, American Stroke Association, Society of Cardiovascular Anesthesiologists, Society for Cardiovascular Angiography and Interventions, Society of Interventional Radiology. Circulation 121:e266-369

3. Vilacosta I, Aragoncillo P, Canadas V, San Roman JA, Ferreiros J, Rodriguez E (2009) Acute aortic syndrome: a new look at an old conundrum. Heart 95:1130-1139

4. Hayter RG, Rhea JT, Small A, Tafazoli FS, Novelline RA (2006) Suspected aortic dissection and other aortic disorders: multidetector row CT in 373 cases in the emergency setting. Radiology 238:841-852

5. Shiga T, Wajima Z, Apfel CC, Inoue T, Ohe Y (2006) Diagnostic accuracy of transesophageal echocardiography, helical computed tomography, and magnetic resonance imaging for suspected thoracic aortic dissection: systematic review and meta-analysis. Arch Intern Med 166:1350-1356

6. Fleischmann D, Mitchell RS, Miller DC (2008) Acute aortic syndromes: new insights from electrocardiographically gated computed tomography. Semin Thorac Cardiovasc Surg 20:340-347

7. Roberts WC (1981) Aortic dissection: anatomy, consequences, and causes. Am Heart J 101:195-214

8. Lesauskaite V, Tanganelli P, Sassi C et al (2001) Smooth muscle cells of the media in the dilatative pathology of ascending thoracic aorta: morphology, immunoreactivity for osteopontin, matrix metalloproteinases, and their inhibitors. Hum Pathol 32:1003-1011

9. Erdheim J (1930) Medionecrosis aortae idiopathica cystica. Arch Pathol Anat 276:187-229

10. Hagan PG, Nienaber CA, Isselbacher EM et al (2000) The International Registry of Acute Aortic Dissection (IRAD): new insights into an old disease. JAMA 283:897-903

11. Morisaki H, Akutsu K, Ogino H et al (2009) Mutation of ACTA2 gene as an important cause of familial and nonfamilial nonsyndromatic thoracic aortic aneurysm and/or dissection (TAAD). Hum Mutat 30:1406-1411

12. Stanson AW, Kazmier FJ, Hollier LH et al (1986) Penetrating atherosclerotic ulcers of the thoracic aorta: natural history and clinicopathologic correlations. Ann Vasc Surg 1:15-23

13. Svensson LG, Labib SB, Eisenhauer AC, Butterly JR (1999) Intimal tear without hematoma: an important variant of aortic dissection that can elude current imaging techniques. Circulation 99:1331-1336
14. Chirillo F, Salvador L, Bacchion F, Grisolia EF, Valfre C, Olivari Z (2007) Clinical and anatomical characteristics of subtle-discrete dissection of the ascending aorta. Am J Cardiol 100:1314-1319

15. Yamada T, Tada S, Harada J (1988) Aortic dissection without intimal rupture: diagnosis with MR imaging and CT. Radiology $168: 347-352$

16. Ganaha F, Miller DC, Sugimoto K et al (2002) Prognosis of aortic intramural hematoma with and without penetrating atherosclerotic ulcer: a clinical and radiological analysis. Circulation 106:342-348

17. Evangelista A, Mukherjee D, Mehta RH et al (2005) Acute intramural hematoma of the aorta: a mystery in evolution. Circulation 111:1063-1070

18. O'Gara PT, DeSanctis RW (1995) Acute aortic dissection and its variants. Toward a common diagnostic and therapeutic approach. Circulation 92:1376-1378

19. Neri E, Capannini G, Carone E, Diciolla F, Sassi C (1999) Evolution toward dissection of an intramural hematoma of the ascending aorta. Ann Thorac Surg 68:1855-1856

20. Nienaber CA, Sievers HH (2002) Intramural hematoma in acute aortic syndrome: more than one variant of dissection? Circulation 106:284-285

21. Erbel R, Alfonso F, Boileau C et al (2001) Diagnosis and management of aortic dissection: Recommendations of the Task Force on Aortic Dissection, European Society of Cardiology. Eur Heart J 22:1642-1681

22. Sueyoshi E, Matsuoka Y, Imada T, Okimoto T, Sakamoto I, Hayashi K (2002) New development of an ulcerlike projection in aortic intramural hematoma: CT evaluation. Radiology 224:536-541

23. Vilacosta I, San Roman JA, Ferreiros J et al (1997) Natural history and serial morphology of aortic intramural hematoma: a novel variant of aortic dissection. Am Heart J 134:495-507

24. Ide K, Uchida H, Otsuji H et al (1996) Acute aortic dissection with intramural hematoma: possibility of transition to classic dissection or aneurysm. J Thorac Imaging 11:46-52

25. Winnerkvist A, Lockowandt U, Rasmussen E, Radegran K (2006) A prospective study of medically treated acute type B aortic dissection. Eur J Vasc Endovasc Surg 32:349-355

26. Murray CA, Edwards JE (1973) Spontaneous laceration of ascending aorta. Circulation 47:848-858

27. Kaplan M, Yapici F, Erkan M, Sargin M, Demirtas MM (2002) Ascending aortic dissection without intimal tear: a case report. Heart Surg Forum 5:E39-E41

28. Kouchoukos NT, Murphy MC (2009) Intramural hematoma associated with an intimal tear. J Am Coll Surg 209:284

29. Timperley J, Banning AP (2003) Prognosis of aortic intramural hematoma with and without penetrating atherosclerotic ulcer: a clinical and radiological analysis. Circulation 107:e63

30. Cho KR, Stanson AW, Potter DD, Cherry KJ, Schaff HV, Sundt TM 3rd (2004) Penetrating atherosclerotic ulcer of the descending thoracic aorta and arch. J Thorac Cardiovasc Surg 127:1393-1399

31. Davies RR, Goldstein LJ, Coady MA et al (2002) Yearly rupture or dissection rates for thoracic aortic aneurysms: simple prediction based on size. Ann Thorac Surg 73:17-27

32. Booher AM, Eagle KA (2011) Diagnosis and management issues in thoracic aortic aneurysm. Am Heart J 162:38-46.e1

33. Debakey ME, Henly WS, Cooley DA, Morris GC, Crawford ES, Beall AC (1965) Surgical management of dissecting aneurysm of dissecting aneurysms of the aorta. J Thorac Cardiovasc Surg 49:130-149

34. Agmon Y, Khandheria BK, Meissner I et al (2003) Is aortic dilatation an atherosclerosis-related process? Clinical, laboratory, and transesophageal echocardiographic correlates of thoracic aortic dimensions in the population with implications for thoracic aortic aneurysm formation. J Am Coll Cardiol 42:1076-1083

35. Reed D, Reed C, Stemmermann G, Hayashi T (1992) Are aortic aneurysms caused by atherosclerosis? Circulation 85:205-211 